This item was submitted to Loughborough's Research Repository by the author.

Items in Figshare are protected by copyright, with all rights reserved, unless otherwise indicated.

\title{
Parenting experiences in elite youth football: a phenomenological study
}

PLEASE CITE THE PUBLISHED VERSION

http://dx.doi.org/10.1016/j.psychsport.2014.05.004

\section{PUBLISHER}

(c) Elsevier Ltd

\section{VERSION}

AM (Accepted Manuscript)

\section{PUBLISHER STATEMENT}

This work is made available according to the conditions of the Creative Commons Attribution-NonCommercialNoDerivatives 4.0 International (CC BY-NC-ND 4.0) licence. Full details of this licence are available at: https://creativecommons.org/licenses/by-nc-nd/4.0/

\section{LICENCE}

CC BY-NC-ND 4.0

\section{REPOSITORY RECORD}

Clarke, Nicola J., and Chris Harwood. 2014. "Parenting Experiences in Elite Youth Football: A Phenomenological Study". Loughborough University. https://hdl.handle.net/2134/17017. 


\section{Parenting Experiences in Elite Youth Football: A Phenomenological Study}

\section{Abstract}

Objectives: The purpose of this study was to explore the experiences of parents of elite specializing stage youth footballers.

Method: A descriptive phenomenological approach guided the study design. Data from interviews with five mothers and five fathers of youth players registered to English football academies were analysed using descriptive phenomenological analysis (Giorgi, 2009).

Findings: Three essences characterized the phenomenon of being a parent of an elite youth footballer: parent socialization into elite youth football culture; enhanced parental identity; and increased parental responsibility. Parents’ socialization into the football academy culture was facilitated by their interaction with coaches and parent peers, highlighting the social nature of parenting. Being the parent of a child identified as talented meant that parents experienced enhanced status and a heightened responsibility to facilitate his development.

Although parents were compelled to support their son in football, their instinct to protect their child meant they experienced uncertainty regarding the commitment required to play at an academy, given the potential for negative consequences. Together, these findings illustrate that parents experienced a transition as their son progressed into the specialization stage of football. We postulate that formal recognition of a child as talented contributed to this transition, and that knowledge of sport and perception of the parent-child relationship shaped how parents adapted.

Conclusions: This study provides a new way of understanding the psychological phenomena of parenting in elite youth football. Implications for practitioners working with parents in sport are provided.

Key words: Parents, youth sport, soccer, qualitative, socialization, career transition. 


\section{Parenting Experiences in Elite Youth Football: A Phenomenological Study}

The sport parenting literature to date has been dominated by research that has sought

to identify the "optimal” behaviours for parents that if adopted will result in positive child outcomes such as higher enjoyment, reduced anxiety and successful progression in sport (e.g. Babkes \& Weiss, 1999; Bois, Lalanne \& Delforge, 2009; O’Rourke, Smith, Smoll \& Cumming, 2011; Wuerth, Lee \& Alfermann, 2004). A focus on parental behaviours has made an important contribution to understanding the role of parents in youth sport, but has often overlooked the social and cultural context in which these behaviours occur.

The influence of contextual factors on parents’ behaviours in sport has previously been identified. For example, Dorsch, Smith, and McDonough (2009) illustrated that parents' behaviour, cognition and affect changed in response to their child's participation in sport, through an interactive process involving the agency of the parent, the influence of the child and the social context. The authors proposed that further research into how these features interact, and specifically parents' responsiveness to their child and the influence of the sport setting, is required to enhance theoretical understanding of parents' socialization experiences in sport.

Qualitative methods are particularly suited to exploring the experiences of parents and allow for the personal and contextual aspects of a phenomenon to be examined. However, only a limited number of studies have thus far investigated parental experiences in sport, meaning parents’ voices in research are currently underrepresented. As Knight and Holt (2013) note, although it is important not to discount children’s experiences, understanding how parents interpret and make sense of their child's sport participation can identify ways in which parents' experiences can be enhanced. For example, in-depth descriptions of how a parent experiences their role can encourage coaches and practitioners to empathize with the demands of being a sport parent (Harwood, Drew \& Knight, 2010; Harwood \& Knight, 
51 2009a, 2009b). Furthermore, Wiersma and Fifer (2008) recommended that theoretical links between parent and child behaviours should be supplemented with research that seeks to understand why or how parents adopt certain behaviours. Therefore, the purpose of the present study was to explore the experiences of parents in elite youth football.

\section{Parental Experiences in Youth Sport}

In one of the few studies that cite giving parents a voice in the purpose of their research, Wiersma and Fifer (2008) held focus group interviews to understand parental involvement in youth sport from the parent's perspective. Parents described the satisfaction they gained from the increased opportunity to interact with their child and the vicarious experience of observing their child learning, enjoying, and being successful in sport. The difficulties parents faced included providing effective support for their child in challenging situations (such as dealing with injury or a lack of motivation) and helping young athletes cope with the demands of their sport. This study highlighted how children can influence parents’ experiences in sport; a finding which has been supported by Knight and Holt (2013), who identified that children's performances, on-court behaviours and emotional reactions to matches affected parents' experiences of watching junior tennis tournaments, and were in fact a source of stress for some parents (Harwood \& Knight, 2009a, 2009b). These exploratory studies point toward the significance of parent-child interactions in sport settings, but did not explicitly examine parents' experiences of this relationship within the scope of their research. Dorsch et al. (2009) described the parent-child relationship as fluid and dynamic, because it can be enhanced from being in the sport environment, but also encounter friction. Frequent or unresolved conflict between parents and young athletes can lead to strained relationships and negative consequences in later years, such as perceived parental pressure and conditional support (Lauer, Gould, Roman \& Pierce, 2010a). Therefore, a better understanding of parent-child relationships can help to identify ways to facilitate positive 
experiences for all those involved. However, parent's experience of interaction and their relationship with children in sport settings has to date received limited attention.

Watching a child participate in a sport event can be an emotional experience for parents, increasingly so as parents invest more in their child's sport over time (Dorsch et al., 2009). Holt, Tamminen, Black, Sehn, and Wall (2008) observed that parents' emotions at youth soccer matches were intensified by contextual factors including crowd segregation, sideline disputes and the game situation, which in turn influenced the nature of their sideline comments. Similarly, from an analysis of parents' accounts of "a time when you became angry during a sport event that your child was participating in”, Omli and LaVoi (2012) highlighted that parents (of athletes aged 5 to 19 years) experienced anger in situations when behaviours of the coach, referee, athlete or other parents were perceived to be unjust, uncaring or incompetent. These studies suggest that interaction with significant others and situational triggers can influence parents' behaviour and emotions when watching their child perform.

Moreover, the characteristics of the sport context can contribute to parents' experience of stress. Parents of youth football and tennis players in the specializing and investment stages of sport (Côté, 1999) described encountering organizational stressors such as selection policies, competition formats and heavy time and financial demands (Harwood et al., 2010; Harwood \& Knight, 2009a). In contrast to grassroots sport, where the parent and coach role may commonly overlap (Dorsch et al., 2009; Wiersma \& Fifer, 2008), parents in elite sport settings also experienced stress from a perceived lack of feedback, communication and respect from coaches (Harwood et al., 2010; Harwood \& Knight, 2009a). This illustrates how parents' relationships with coaches can change as children progress in sport, and demonstrates the need to understand parental experiences within specific cultures, given each sport's uniqueness (Dorsch et al., 2009; Lauer et al., 2010a; Lauer, Gould, Roman \& Pierce, 
101

102

103

104

105

106

107

108

109

2010b).

Dorsch et al. (2009) recommended that future parenting research include the community sport context within its focus. Although studies have highlighted contextual influences on parents' experience of watching their child perform in competition (e.g. Holt et al., 2008; Omli \& LaVoi, 2012) and of stress (Harwood et al., 2010; Harwood \& Knight, 2009a), a broader investigation of the influence of the sport setting on parents' experiences presents a gap in the literature.

In order to address the limitations of the existing literature, and enhance understanding of the influence of the social and cultural context on parenting in youth sport, a phenomenological approach was employed in this study. Phenomenology rejects the subjectobject dualism that underpins traditional positivist and post-positivist research, and instead seeks to explore experience and how the world appears to people through a focus on the intentional relationship between a person's consciousness and the object to which it is directed (Giorgi, 2009). In doing so, phenomenology places social interaction and the cultural context at the heart of the research endeavour. Accordingly, this research aims to explore how parental experiences are shaped by the personal, social and cultural context of elite youth football.

\section{Methodology and Methods}

\section{The Sport Context}

The youth football talent development system in England is managed by a network of professional football clubs, which offer intensive training and competition programmes to male players aged 8 to 18 years who are identified as talented. These elite players will attend a football academy; a training environment which aims to produce players for the professional game (The Premier League Elite Player Performance Plan, 2011). Parents of players in the initial phase of the specializing stage (aged 8 to 11 years; as defined in 
126 Harwood et al., 2010) help players to manage the transition from grassroots to elite academy

127 level football, and are highly involved through the considerable weekly training and

128 competition commitment that academies demand. Players are expected to train between five

129 and eight hours per week, typically structured over two weekday evenings and both weekend

130 mornings (The Premier League Elite Player Performance Plan, 2011). As players can live up

131 to a maximum of one hour travel time from the football club, parents are responsible for

132 transporting their child to training and matches. Harwood et al. (2010) identified a range of

133 stressors that specializing stage parents in football described experiencing, arising from

134 academy expectations, practices and communication. Parents struggled to accept academy

135 practices which prioritized player development over winning, and felt that they received

136 inadequate feedback on their child’s progress and limited appreciation of their role from

137 coaches. Given the significance of the transition to academy football for parents and players,

138 and the potential for parents to experience stress, the present study explored the experiences

139 of mothers and fathers of specializing stage footballers.

\section{Participants}

Parents of boys registered to an elite football academy aged between 8 and 11 were recruited from three English professional football clubs. Five mothers and five fathers aged between 38 and 56 participated in the research $(M=43.8)$. Three of the mothers were single-

144 parents; all other participants were in two-parent families. Parents described their ethnicity as

145 White British ( $n=8)$, Black British $(n=1)$ and Spanish Bengali $(n=1)$. Parents had between

146 two and four years experience of their son playing at a professional football club academy.

147 Further demographic details can be found in the Supplementary Material.

148 Research Design and Philosophical Assumptions

A descriptive (or empirical) phenomenological research design was followed in this

150 study; a branch of phenomenology developed by Giorgi (Giorgi, 2009; Giorgi \& Giorgi, 
151 2008a, 2008b). Rooted in the original philosophy by Husserl who called for 'a return to

152

153

154

155

156

157

158

159

160

161

162

163

164

165

166

167

168

169

170

171

172

173

174

175

things themselves', descriptive phenomenology seeks to provide rich, textured descriptions of phenomena, to understand more about how the world is perceived and what it means to people. Using this approach, we set out to describe participants' lived-experiences (meaning existence as it is experienced through the intentional relationship between a person's consciousness and phenomena) using first-person accounts. However we make no claims as to whether these are a 'true’ reflection of experience, adopting instead a relativist epistemological position that assumes knowledge is constructed. This study commits to the multiple and constructed nature of reality and assumes peoples' experiences, and the meanings people attach to those experiences, are individually interpreted and shaped by their social, cultural and historical backgrounds.

\section{Methods}

To become familiar with academy context, the first author arranged ten visits to one academy to talk to parents and coaches, and observe training sessions and match days. During the visits, which were typically for two hours, the first author sat with parents in the cafeteria area, engaging in informal conversations, and joined parents to watch players train or compete from the side line. The role of an "observer as participant” was adopted, where the researcher primarily observes without being directly involved, but participates in the setting through their interaction with others (Gold, 1958). Observations were recorded in a research diary and later used as a tool to reflect on any pre-conceptions that had formed as a result of exposure to the setting.

Ethical approval was obtained from the university’s ethics committee and all participants gave informed consent. Following initial information meetings held at the three academies, parents interested in being involved were asked to contact the first author.

Selection was then guided by maximum variation sampling. Variation between participants is 
beneficial in descriptive phenomenological work, as aspects of an experience that are common to a group of people and those which are unique to individuals can be highlighted.

Accordingly, mothers and fathers, one and two-parent families and parents from the three different academies were sampled, enabling a range of parental experiences to be captured. Semi-structured phenomenological interviews were held with individual parents over a three-month period. The purpose of a phenomenological interview is to gather "as complete a description as possible of the experience that a participant has lived through" (Giorgi, 2009). In designing the interview schedule (see Supplementary Material), care was taken to ensure the questions were open rather than directive, so to encourage the participants to present their experience as it was relevant to them. Questions such as "can you describe a typical week for you?", "what is your role as a parent on match days?” and "what does being a parent of a young footballer mean to you?” guided discussion, and probes were used to encourage participants to provide more detailed descriptions. Participants were also able to lead and shift the conversation, which meant that individuals could introduce topics that were meaningful to them beyond those discussed through the interview guide. Pilot interviews were held with three parents of youth footballers to trial the questions and technique. Parents were interviewed once, by the first author. Interviews lasted between 68 and 106 minutes $(M=85)$ and were held at academy training grounds, parents' houses and the university. Interviews were audio-recorded and transcribed by the first author. O'Connell and Kowal (1999) recommend that a transcription system should match the purpose for which it is required, and that only those elements of interviews that will be analysed need to be transcribed. Therefore, all verbal talk was transcribed verbatim into a play-script format. To provide confidentially to participants, culturally appropriate pseudonyms were chosen for each parent and for any football club or person mentioned by name in the interviews.

\section{Data Analysis}


phenomenological approach, and employed the concepts of bracketing, reduction and a

search for essences. Throughout the research process, bracketing was used to set aside the

natural attitude (the everyday view and taken for granted assumptions about the world) and

remain open to things as they appeared. This entailed the first author being aware of, and

aiming to put aside as much as possible, presuppositions of scientific theories, knowledge and explanations, and her personal views and experiences (Ashworth, 1996). Performing the

reduction enabled the first author to conduct analysis from within an open phenomenological attitude, by staying meticulously close to data, treating all data with equal significance and ensuring that participants’ descriptions were still recognizable following any interpretation. participants' descriptions. The data was then attended to with a broad psychological lens and "sensitivity to the implications of the data for the phenomenon being researched" (Giorgi, 2009 p.128). Next, meaning units were established, by marking the transcript each time the researcher interpreted that a change in psychological meaning occurred, to help make the

216 lengthy descriptions more manageable. No data was omitted from this process in order to avoid privileging some over others. An analysis matrix was completed for each participant to make sense of the data and provide a trail of how meaning units were identified for the second author. Each meaning unit was described in more neutral language so that by lifting the data from the situation-specific details, the psychological significance could be clarified.

221 Units were then transformed into language representing the psychological meaning of the data in order to "render the implicit explicit” (Giorgi \& Giorgi, 2008b p. 45). No explanations for meanings were offered at this stage as the researcher sought to remain close to the experience as the participants described it. An example analysis matrix is provided in the

225 Supplementary Material. 
Each participant's fully transformed description of experience was analysed and

227

228

229

230

231

232

233

234

235 interpreted individually, before comparing to others to identify the essential, invariant qualities of experience - or essences of the phenomenon, which "make a phenomenon what it is and without which the phenomenon could not be what it is” (van Manen, 1990, p.107). Interview transcripts were explored further using the phenomenological technique of imaginative variation (Giorgi, 2009; Moustakas, 1994). This involved actively considering the data from different angles to identify essences which continued to have the same psychological meaning, even when the context of the data was imagined to be different. For example if the gender of a parent was imagined to be the opposite, did the meaning of the experience change? Imagining variances in the data requires practice to fully exploit the interpretative benefits; however the first author strived to use the technique to the best of their ability. Individual structure statements were composed describing the meanings of participants' experiences which assisted in the final step; presenting the overall general structure and essences of the experience of being a parent of an elite specializing stage footballer.

\section{Enhancing Research Quality}

Following a relativist approach to judging qualitative research, we used the characteristics of sensitivity to context, reflexivity, rigor and coherence to enhance the quality of our research (Sparkes \& Smith, 2009; Tracy, 2010; Yardley, 2000). To achieve sensitivity to the social and cultural context of academies, the first author spent time in the field, observing participants, to become familiar with the day-to-day activities of an academy, the idiosyncratic language of football, and the relationships between people within the setting. To enhance reflexivity, the first author participated in a bracketing interview, conducted by a colleague not involved in the research project itself but with experience of qualitative investigations. The purpose of the 60 minute interview was to reveal any underlying biases or 
opinions that could potentially influence the data collection and analysis process. Rather than aim to remove these influences from the research process, the first author was able to consciously check that these underlying suppositions were not directing the interviews or the analysis in a particular direction. A research diary was also kept for the duration of the project to record reactions to the interviews, reflections on the data analysis process and initial interpretations. Many diary entries described 'gut-instincts' to the data being transcribed or analysed. To assist in the bracketing process, these initial thoughts were set aside and analysed interview transcripts were re-read to see if any alternative interpretations could be formed.

Furthermore, the individual descriptions of participants' experiences and the psychological interpretations, as well as emerging themes and the structure of essences were shared with the second author. Experienced in working in football as an applied sport psychologist, parent/coach educator and researcher, the second author strengthened the analysis process by offering additional and sometimes alternative meanings to the descriptions of parents' lived-experiences. Following guidance for evaluating phenomenological projects (Cresswell, 2007 Norlyk \& Harder, 2010) we have attempted to articulate an understanding of the key theoretical tenets of phenomenological psychology and how they have been implemented. Through doing so, it is hoped the reader can assess whether this study achieves coherence; a meaningful fit between the research question, methodology, analysis and interpretation of findings (Tracy, 2010; Yardley, 2000).

\section{Findings and Discussion}

From our interpretations, we suggest that the experience of being a parent of an elite youth footballer constituted three essential features; parent socialization into the elite youth football culture, enhanced parental identity and increased parental responsibility. Though the essences (and the subthemes which support them) characterize the phenomena, there were 
individual variations in the psychological meanings that parents attached to their experiences, which are also outlined in order to give as full a description possible. In this sense, however, it is perhaps more appropriate to view the findings as expressions of the nature of existence rather than essential structures of being.

\section{Parent Socialization into Elite Youth Football Culture}

Jane: It's a big transition (.) For him and for me ${ }^{1}$.

The parents in this study described the process of adjusting to the different social norms and behavioural expectations associated with the academy football culture. The demands on players to attend a higher amount of training meant that parents' overall commitment to football increased. They were required to invest significant time and money, and organize working hours, childcare and transport to enable their sons to play at an academy. As a result, parents described their decision to support their son’s football as "a life choice for me” or "it’s like my hobby now". However, their involvement in coaching and matches decreased, as the professional status of the coaches in an academy emphasized that parents should "let the coaches coach”. This meant parents were expected to encourage but not instruct players, remain quiet during matches and refrain from questioning coaching decisions. Observing other parents reinforced that giving instructions from the sideline was not an acceptable way of behaving and that parents would "look silly if you did”. These norms for sideline behaviours restricted parents from performing parenting in the way they had previously at grassroots level. By conforming to new rules, parents’ movement was limited to certain spectator areas and their capacity to comment or gesture minimized. Before, football had been a setting where parents could enact involved parenting, as one mother reflected: Jane: You go to those [grassroots] matches and, if the boys want to come over and

\footnotetext{
${ }^{1}$ Transcription notation: (.) indicates a pause; - indicates cut-off speech or self-interruption
} 
have their shoe laces tied up they could run over and do that, and if you had the drink they could come over to you. Here it's, no. Your job is to stand on that sideline and not talk to the boys or interfere with the boys. Which is absolutely right (.) but it is still a loss of control so (.) a bit difficult.

Limiting parenting to certain spaces meant parents experienced a diminished sense of agency and, at times, frustration from not being able to give their son the technical advice they believed would help him be successful.

Dorsch et al. (2009) has previously identified the community sport context as a potential moderator of parent socialization into sport. Our findings suggest that it is interaction with others within the sporting environment that can socialize parents into particular roles. This essence reflects a central concept of phenomenology, that all experience is relational (e.g. Husserl, 1931; Merleau-Ponty, 1962). In academies, parents learnt about what constituted appropriate behaviours in this setting through their interaction with coaches and other parents, which facilitated parents' socialization into the elite football culture. Although, how parents interpreted and responded to these expectations varied, and is described below.

Negotiating power and responsibility with coaches. Coaches reinforced that player development was their sole responsibility by limiting communication to parents once players were signed to a club and offering less feedback on children’s performances. Consequently, approaching coaches to ask questions outside of formal appraisal meetings was construed as “interfering” by parents. Parents had to adjust to coaches having responsibility for their son’s football development, but welcomed or resisted this transference of power to different extents. Some parents were happy to relinquish responsibility as they saw coaches as experts, trusted them and felt they were good role models for their son. 
here, three times a week, you have complete control over whatever you do with that lad in terms of his coaching, discipline, everything else. I'd sign it and, you know, I just don't think that parents should interfere. I think they should just leave it. Parents began to experience pressure to comply with coach requests such as attending extra training sessions during school holidays. Although these were optional, they felt that non-attendance would be negatively perceived by coaches. At times, parents acquiesced to the demands placed on players to gain approval from coaches and avoid jeopardizing their son's place in the squad. However, other parents found letting go of their previous involvement more difficult.

Andy: I think that is one of the hardest things you have to come to terms with when you bring them here is that, all the things you believed in, and I'm saying this from someone who knows about football is that, you've done your bit. And it's now for somebody else to do it and, they've got to polish a rough diamond. And you probably won't take any- you won't get any of the credit for quite a bit of the formative stuff. You know, because although he’s here on his own ability, if I'd have let him play the PlayStation and done nothing with him then he wouldn’t be here.”

And some even challenged the coaches' role in player development. For example one father rejected the authority of the academy staff, asserting his control of the situation by maintaining that he - not the coaches - would decide when his son would leave the academy. James: I just think people once they sign a contract think they’re obliged. You know they have to- No you don't! You have a choice you can always walk out. Nicola: You do have a choice yeah. James: Now one parent this year who walked out, they want compensation for him. So they try and make it difficult for you. But, if I'm going, we can go at any time we want, Academy A can't stand in my way. Because the power is with us. They make 
you believe they've got the power. They don’t! We can just say we stop, we don’t want to do it no more.

Parents who felt uneasy about the transference of responsibility to coaches described

how they were in the process of learning to trust the coaches' methods of developing players.

Often, it was fathers who had previous experience of coaching youth football who described struggling to accept the coach's role the most and were more likely to question decisions.

Over time parents became more accepting of coaching practices, however some felt the reduction in communication once players were signed prolonged this adjustment. control and being asked to trust coaches formed an early phase in parents' socialization into elite youth sport culture. In youth football, parents accepted or contested the transference of responsibility to coaches to different degrees, and our findings illustrate how parents can be marginalized through a reduction in communication from coaches, a pressure to conform and an increased sense of need for approval from coaches.

Parent peer relationships. Parents regularly interacted with other mothers and fathers of youth footballers and these relationships performed several important functions. Parents new to the academy setting were able to seek advice from peers whose sons had been at the academy longer, as more experienced parents could explain what could be expected in their first season, help interpret unfamiliar coaching practices and offer feedback on their son’s performances. Parents drew support from the friendships they made at academies as other parents offered help with transport duties and an empathetic understanding of the stress they experienced that friends outside of football could not provide. Yet the temporal nature of these friendships was recognized, as parents anticipated that relationships would not continue

373 if their son no longer played at an academy. 
because it is a nice atmosphere here and because you all get on - you knowing that actually it's like a group of friends you're gonna lose.

Parent peers also provided a target for social comparison. Parents were judged

positively by others when they were realistic about their son's chances of becoming a

professional, knowledgeable about football development and seen not to push their sons. In contrast, parents were compared less favourably when they expressed ambitions for their sons to "make it", were seen to pressure their child to play, or were viewed as parents of less talented players. Comparing themselves and their sons to others in an academy helped to affirm parents’ own identity as a realistic parent, who knew how best to support their son’s development.

James: You sort of get rated as a parent as well. If your kid's up there, you're one of the good parents you know and one of the parents- honestly! Your, your kids down here, you know you see the parents’ body language, slinks a bit and you know (.) god streuth yeah, it's pretty sad.

Phil: I think as parents you learn, erm, certainly you have to learn by your mistakes along the way about maybe how you behave at the side of a pitch, how you behave in the car. And I watch other parents and quite often I'll look at 'em and think 'crikey, I can’t believe that used to be me'.

Relationships with parent peers have been identified as a common feature of youth sport parenting experiences and previous research has highlighted that parents valued the support and social networking offered by peers, but could experience feelings of exclusion when cliques form (Dorsch et al., 2009; Knight \& Holt, 2013). The findings in our study extend existing knowledge by describing how these relationships not only functioned as supportive, but helped to socialize parents into the sport culture by providing advice in the absence of information from the academy. In addition, peers were used by parents to evaluate 
400

401

402

403

404

405

406

407

408

409

410

411

412

413

414

415

416

417

418

419

420

421

422

423

424

their own parenting effectiveness. This may in part explain Knight and Holt’s (2013 p. 8)

finding that cliques could develop in youth tennis between "parents of the better players".

\section{Enhanced Parental Identity}

Parents felt that their child's identification as a talented footballer reflected positively

on their own identity as a parent. Many recounted how they were the first to recognize that

their son had potential in sport; a view that was reinforced when parents started to be approached by professional club scouts and coaches. Players often received multiple offers to join academies before committing to one club, and their football ability was praised highly in the process. Having a child that was good at football meant that parents felt they had been successful in parenting.

Sarah: I'm just really proud because I think umm (.) I've done it all myself and that's because when I (.) I’ve been a single parent for a long time and it was me, I feel like it was me that got Tyler involved in all of his football. Even though I'm not a football person, I arranged the meeting, I got in touch with somebody and I was taking himhis dad only takes him to his matches, he doesn’t get involved with any of the training. So I've sort of nurtured him and encouraged him and I feel as though I've done a good job and it makes me proud. I'm proud of him and I'm proud of myself because I’ve done it myself.

The more intensive schedule and greater emphasis on football in their lives enhanced parents' relationships with their sons. The experience of being part of a professional club, sharing a passion for football and travelling to and from training and matches together, increased the sense of closeness in the relationship.

Tom: It is very interesting how the club in some ways intensifies the relationship with your child. I suppose we're together so much, we travel so much, we talk so much, we, and when we're not here, we're watching the first team. We even go to away 
games. We're very tight.

Peter: He does really appreciate me taking him here and he can’t thank me enough, you know, all the time. So he does, so me and Joe really are close because of it. Experiencing closeness with their son through football meant that parents shared in his successes and failures. For example, parents experienced apprehension before coach assessments and reflected glory from their child's achievements, often referring to "when we got signed” or "when we played United”. Coakley (2006) has suggested that the public, measurable nature of youth sport means that the moral worth of parents can be symbolized through their child's sporting success. Certainly, the parents in this study experienced enhanced status from their child's selection to an academy. Consequently we propose that the identification and labelling of young children as talented in sport amplifies the extent to which parents’ identity and personal worth are reflected in their children’s achievements. Parents had learnt that success in academies was measured not by matches won and trophies awarded, but by individual effort and improvement. Often their child who was the superstar in their local team, stood out far less in the academy. Eager to see him succeed in a more challenging environment, their son's performance in football became increasingly more important.

Phil: Because it's football it seems to be life or death, whereas everything else, you know if I had a bad review here for football I'd probably be gutted. But if he had a bad parents evening I’d probably just let it go over me head. And that's completely wrong you know. But it’s taken me time to learn that and understand that. Andy: It's not the be all and end all ... it just feels like that sometimes. This connection to their son's sporting identity led some parents to anticipate experiencing identity disruption if their child was deselected and no longer played at an academy. Helen: There is immense pride that my children play for Academy B and Academy D. 
But with that there comes a fear that if they didn’t play at Academy B would you feel that they had failed all of a sudden if they didn’t get taken on, or they couldn’t play at Academy B and you had to say they played at their local club. And I think, if I'm entirely honest, that might be an issue for me.

Transition out of elite sport that is sudden or involuntary can be an emotionally turbulent and disruptive experience for athletes (Blinde \& Stratta, 1992; Wippert \& Wippert, 2010). Our findings suggest that parents may also experience identity loss following a child's unexpected exit from sport, and highlights an area worthy of further investigation.

\section{Increased Parental Responsibility}

In addition to an enhanced identity, parents experienced an increased sense of responsibility toward their son and drew on societal expectations for parenting to make sense of this responsibility, adjusting their behaviours accordingly (in various ways, described below). This was not, however, a straight forward adjustment, as although parents wanted to help their sons succeed in football, they were also acutely aware of the potential for negative consequences of playing in an elite academy environment. This inherent tension between supporting and protecting their child meant that parents experienced uncertainty and at times, interpreted the meaning of academy football differently.

Duty to facilitate development. Being the parent of a child recognized as talented in sport meant that parents experienced a heightened sense of responsibility to facilitate their son's development in football and help him realize his potential.

Hannah: I've stressed to Lucas (.) I, I do this for you, this is an investment, and I don't mind doing this because if you have a talent it's my duty to kind of let you see if you can do something with that talent. . . . I will do for him exactly what I'll do for my daughter as well. If they have a talent, I will do everything I can for them to succeed at what they enjoy in life or what they're good at in life. 
475 Yet parents had to manage this additional responsibility in line with the expectation to be a 476 realistic football parent, which they learnt about through comparisons to their peers. This 477 meant negotiating a balance between supporting, but not pushing, their child to succeed. In fulfilling this responsibility, parents often adjusted their own behaviours in response to their child, such as waiting until their son was ready to discuss matches 480 afterwards and recognizing that certain technical feedback was better received by players if it came from coaches. They were keen to follow dietary guidance for players provided by sports nutritionists and closely monitored their child's mood to respond to his needs. Mothers described placing their child's needs above their own; sacrificing their free time and personal relationships in order to support their son's football commitments. This aligns with Wolfenden and Holt's (2005) finding that mothers were happy to give up their social lives and support their child's increasing tennis commitments, in order to ensure their child had access to the best opportunities. However, when mothers lacked experience of football, they experienced uncertainty over the degree to which they should be encouraging their son to practice and focus more on the sport.

Helen: I think because I'm a Mum, I have a, I think I have a slightly different attitude. If he was a girl playing netball, I think I would be a lot more pushy, umm because that was my sport, and I think I’d know a lot more.

Conversely, fathers who had experience of playing or coaching football, explained that they understood the standards required from players at different stages, would recognize whether their son was achieving them and if not, provide instructions on how to improve. Holt et al. (2008) previously demonstrated that knowledge and prior experience of sport affected parents' verbal reactions to their child's performance. In youth football, fathers saw their knowledge as enabling them to fulfil their parental responsibility - to help their child achieve 499 his potential. 

reciprocal nature of the parent-child relationship, and that parents adjust their behaviours in response to their child's temperament, performance and behaviour (e.g. Dorsch et al., 2009; Holt et al., 2008; Knight \& Holt, 2013). Developing this further, when parents in academies found it difficult to read and understand their child's feelings, the intensity of the football experience highlighted this shortcoming, as one father described upon learning that his son was not enjoying football.

Phil: I felt it upset me more than it probably upset him in that somebody else has had to tell me that your son's not enjoying it. And that was his granddad. My dad pulled me to one side and said “do you know how unhappy your lad is?” and I’ve just been on the crest of a wave thinking oh he's enjoying it and he's going to Academy $\mathrm{X}$ and Academy Y, and I'm thinking he's loving it. But then when he's going to his grandparents in the week he’s saying “I don't like this and I don’t like that”. When I found out about it I think you feel a bit (.) a bit of a failure to be honest as a dad that you've not recognized it before. And upset that he's not been able to come to me. meet societal ideas of what constitutes "good parenting” - in this case, the expectation for fathers to develop open and close relationships with their children (Dermott, 2003; Henwood \& Procter, 2003). The social construction of parenting and the influence this has on parentchild relationships has received limited attention in sport psychology, despite authors such as Coakley (2006) and Kay (2009) describing how societal expectations for parents can be lived out in a sport context, and offers a potential direction for future research. were giving their son the best chance of succeeding, as they recognized academies as offering the best opportunity for players to improve and as part of the journey to becoming a 
525 professional adult footballer. This perception compelled parents to continue to support their son in football, to help him reach his full potential. However, if parents reflected that it was unlikely that he would ever be offered a professional contract, their instinct to protect their son meant they questioned whether the commitment required at such a young age to play at an academy was worth it, given the potential for negative experiences. For example, players had less time to socialize with friends outside of football and were often tired from late nights travelling home after training.

Phil: We leave home at 4.00pm to beat the traffic and we usually get down here for about 5:15pm, and it's dark when we leave, it's dark when we get home. He’s asleep for most of the journey home. We get in at 8:45pm. There's times when I've carried him from the car straight to bed and I'm thinking 'what am I doing putting him through this?' It's madness.

Players were also exposed to the potential disappointment of de-selection or "release"

from academy squads. Being released was seen as a constant threat to player's well-being and future in football, as all parents had heard stories of other players and parents who had not coped well with release, or had not expected it. Parents talked about academies as being cutthroat, competitive and ruthless, where only the good enough survive. Consequently parents were torn between the concern that the professional club environment was inappropriate for young children and the perception that academies were the best place to develop players. James: I'm really mixed and ambivalent on academies. You know, because I- I just think it's too early. Telling a kid you're not good enough at nine or ten is unnecessary. They don't need to face the sharp end of the world at that age, you know. It doesn't need to be that at nine or ten. There's no place in the world for that. But if I took Harry out and put him back in the youth team, he really won't have anyone to play with, because the talent's diluted. If I leave him in here he's got to deal with the 
550

551

552

553

554

555

556

557

558

559

560

561

562

563

564

565

566

567

568

569

570

571

572

573

574

pressures that come with this. So they've put you in this either or position, which is poor.

By focusing instead on the meaning of academy football as an opportunity for their son to play in fantastic facilities and learn skills such as discipline, respect and teamwork, parents resolved to carry on taking their son to the academy as long as he was enjoying playing and improving in football. In addition, to try and ensure their son remained realistic and kept football in perspective, parents emphasized the importance of education and encouraged their sons to take part in a variety of sports, recognizing that the numbers of players who would ever become professionals were small.

James: I've gone and educated myself. I’ve looked at the numbers. I've looked at the stats of how many make it and how many don’t. So if I know that and I pull my kid up and tell him all these lies about being a footballer, knowing that actual statistical numbers, that would be a mistake on my part. So I've gotta keep him realistic.

Parents regulated the temptation to look ahead to their son's potential future in football by trying to focus on being in the present and enjoying the experience for as long as it lasted, downplaying the meaning of academy football as a potential route into the professional game. Peter: If he can stay here until he’s at least 14 and learn about life, being a footballer then I'll be very happy. If he's beyond that then I'll be, I'll be ecstatic and that's how I look at it because you can’t (.) I don’t think you can get too hung up about saying well you gotta get to you're 16 because then you've got this and then you can have that. I think you're setting yourself up for a big fall if you do that if I'm honest because there’s only a very small percentage that actually make it . . . I think, keep your feet on the ground and just enjoy the experience while it's happening. Uncertainty about a child's future in sport has been cited as a common stressor among sport parents (Harwood et al., 2010; Harwood \& Knight, 2009a). By reorienting towards the 
575

576

577

578

present and their son's short-term enjoyment from football, parents suppressed their concerns and resolved to help their son as long as he was progressing. This finding demonstrates how parents coped with uncertainty and reflects the temporal dimension of being a parent of an elite youth footballer, often lost in retrospective accounts.

\section{Conclusions}

\section{Phenomenological Interpretations}

Using a phenomenological approach as the theoretical lens for this research - which differs from a traditional cognitive or behavioural view of psychology (Langdridge, 2007) has provided a new way of understanding the psychological phenomena of parenting in elite youth football. The essences which together constituted parents' experiences: socialization into the elite youth football culture; enhanced parental identity; and increased parental responsibility, can be described in phenomenological terms as reflecting fundamental aspects of lived-experience (Ashworth, 2003; Merleau-Ponty, 1962). In particular: relationality (the experience of social relations as lived); selfhood (what the phenomenon means for identity and agency); embodiment (the role of the body in experiencing a phenomenon including emotions); and temporality (the lived-sense of past, present and future).

The significance of relations with coaches and peers to parents' socialization experiences and behaviours in this study illustrates the social nature of parenting. Yet current theorizations of parental influence in sport do not account for social interaction in their explanations. Fredricks and Eccles’ (2004) model, used extensively to underpin sport parenting research (e.g. Bhalla \& Weiss, 2010; Bois et al., 2009; Gould, Lauer, Rolo, Jannes \& Pennisi, 2008), identifies a number of social and cultural demographics that affect parental influence, but does not recognize the influence of relationships or the sport environment. By theoretically conceptualizing parenting as a social process and considering issues of relationality alongside individual characteristics, a more complex understanding of parental 
600 influence may be produced. In particular, the relationship and interaction between parents and 601 children, coaches and peers should be considered in theories which seek to account for 602 parental behaviours in sport.

604 being part of the elite youth football culture shaped this relationship. Through their son's 605 selection to an academy, parents' identity was enhanced and became closely linked to his 606 football participation. Rather than seeing the self as residing within the individual,

607 phenomenology constructs identity as something which is developed through social interaction. As Merleau-Ponty (1962, p.122) explains; “we are literally what others think of

609 us and what our world is”. For the participants in this study, their identity as a parent was

610 formed through their relationship and interaction with their child in the football setting. The embodied aspect of parenting has previously not been explored in sport contexts.

612 Our findings highlight how parents felt restricted when they were unable to perform

613 parenting in the same way they had at grassroots level, as the responsibility for player

614 development shifted to coaches. The tension between the embodied instinct to protect their

615 son from negative experiences, and the desire to ensure their child had access to the best 616 opportunities to develop and improve, meant parents experienced uncertainty. Following

617 Merleau-Ponty’s perspective that “we perceive the world with our body” (1962, p.239) and

618 that "it is precisely my body which perceives the body of another" (1962, p.412), we can

619 view embodied parent-child relationships as a key feature of what it means to be a parent.

620 Exploring the implications of club policies which may restrict parents from parenting is an important area for future research.

623 reflected in how parents recognized the transient nature of their friendships with peers, and

624 how they coped with uncertainty about their child's future in football. By focusing on being 
625 in the present and their son's short-term enjoyment from football, despite the temptation to

626 project to a possible future in which their son becomes a professional player, parents

627 suppressed their concerns. These findings provide insight into parents' experiences, often

628 overlooked in retrospective studies.

\section{Parent Transitions in Sport}

630 Together, the findings of this study suggest that parents experienced a transition as

631 their son progressed into the specialization stage of football. Much has been written concerning young athlete's career transitions in sport and the changing roles and involvement of parents across an athlete’s sport career (e.g. Alfermann \& Stambulova, 2007; Côté, 1999;

634 Lauer et al., 2010b), and yet parents’ own adaptation to these transitions remains relatively unexplored. In football, parents' transition into the specialization stage was associated with a change in identity and an increased sense of responsibility to facilitate their son’s development. We postulate that formal recognition of a child as talented contributed to these changes, and that knowledge and previous experience of sport, together with perception of the parent-child relationship shaped how parents adapted. Further studies are required to understand how transitions in sport are experienced by parents and what constrains or enables parents to adapt successfully. For example, Lally and Kerr (2008) interviewed parents three to five years after their daughters had retired from elite gymnastics, and described how parents experienced a sense of being "lost” in their relationships with their daughter, their

644 partner and their parent peers following the end of their daughter's sporting career, and that 645 most parents were ill-prepared for the transition. Understanding how parental identity 646 changes following a child's exit from sport may therefore be of interest, given the potential 647 for identity disruption. 
650 faced with an increased sense of responsibility for their son's development, the parents in our study relied upon societal constructions of parenting and subjective comparisons to peers to make decisions and judge whether they were parenting effectively. Informing parents about player development pathways (including exit routes) and expected performance and practice

654 levels across different stages is essential. In sports where performance criteria are not clearly

655 defined, explaining how young athletes will be judged may help to reduce uncertainty around children's future in sport. We would advise however that in elite settings, parents are encouraged to measure their child's success in terms wider than just whether their child keeps a place on a particular squad or team. The coach's role in this communication to parents is imperative, although our findings question the extent to which coaches are aware of their role in shaping the experiences of parents. we suggest that parents may benefit from more individualized advice and guidance. Fathers experiencing difficulty adjusting to a reduction in responsibility for their child’s football development, or mothers with a lack of access to experiential knowledge of playing men’s football may require different forms of support. The latter need for support is particularly pertinent given the number of single mothers in our sample. Other parents who can empathize with the emotional experience of parenting in youth sport may be best placed to explain to newer parents how they adapted to the different culture.

\section{Limitations} coveted and academy squads are assessed regularly by coaches by performance standards that are not clearly articulated to parents, means that caution should be taken with applying our

673 findings to other sports. Perhaps coach evaluations of children's performances would be less

674 important to parents in a sport where young athletes' progress can be tangibly measured 
675 through grades or ranking points, although this system comes with its own challenges, such 676 as increasing the emphasis on winning (Knight \& Holt, 2013). This study may also be limited

677 in that despite seeking variation in our sample, the participants all volunteered to take part in 678 interviews and may share common characteristics different to those parents who declined or 679 did not respond to research invitations. More research in different populations of parents of 680 elite youth footballers may help to further delineate the essences of experience. Finally, this 681 study did not seek to understand how individual variations in parental experience influenced

682 child outcomes. In order to comprehensively understand parental influence in sport, 683 children's perceptions, interpretations and experiences of being parented must also not be 684 forgotten in research.

685 Phenomenological research in other contexts may further contribute to our 686 understanding of the complex nature of parent-child relationships in sport and encourage this 687 vital area of research to move beyond defining parents by a checklist of positive and negative 688 behaviours, and instead highlight how practitioners can understand parents and help them to 689 adjust to the demands of being a sport parent.

\section{Acknowledgements}

691 This research was supported by the English Football Association as part of its

692 Psychology for Football research strategy. We also would like to thank the reviewers for their 693 thoughtful and constructive suggestions during the peer-review process. 


\section{References}

695 Alfermann, D., \& Stambulova, N. (2007). Career transitions and career terminations. In G.

696 Tenenbaum, \& R. C. Eklund (Eds.), Handbook of sport psychology (3rd ed., pp. 712736). Hoboken, NJ: John Wiley \& Sons, Inc.

Ashworth, P. (2003). An approach to phenomenological psychology: The contingencies of the lifeworld. Journal of Phenomenological Psychology, 34(2), 145-156.

Ashworth, P. (1996). Presuppose nothing! The suspension of assumptions in phenomenological psychological methodology. Journal of Phenomenological Psychology, 27(1), 1-25. doi: 10.1163/156916296X00014

Babkes, M. L., \& Weiss, M. R. (1999). Parental influence on children's cognitive and affective responses to competitive soccer participation. Pediatric Exercise Science, 11(1), 44-62.

Bhalla, J. A., \& Weiss, M. R. (2010). A cross-cultural perspective of parental influence on female adolescents' achievement beliefs and behaviors in sport and school domains. Research Quarterly for Exercise and Sport, 81(4), 494-505. doi: 10.1080/02701367.2010.10599711

Blinde, E. M., \& Stratta, T. M. (1992). The "sport career death" of college athletes: Involuntary and unanticipated sport exits. Journal of Sport Behavior, 15(1), 3-20.

Bois, J. E., Lalanne, J., \& Delforge, C. (2009). The influence of parenting practices and parental presence on children's and adolescents' pre-competitive anxiety. Journal of Sports Sciences, 27(10), 995-1005. doi: 10.1080/02640410903062001 Studies, 25(2), 153-163. doi: 10.1080/02614360500467735 
719 Cresswell, J. W. (2007). Qualitative inquiry and research design: Choosing among five approaches (2nd ed.). Thousand Oaks, CA: Sage Publications Ltd.

721

722

723

724

725

726

727

728

729

730

731

732

733

734

735

736

737

738

739

740

741

742

743

Dermott, E. (2003). The 'intimate father': Defining paternal involvement. Sociological Research Online, 8(4). Retrieved from http://www.socresonline.org.uk/8/4/dermott.html.

Dorsch, T. E., Smith, A. L., \& McDonough, M. H. (2009). Parents' perceptions of child-toparent socialization in organized youth sport. Journal of Sport and Exercise Psychology, 31(4), 444-468.

Fredricks, J. A., \& Eccles, J. S. (2004). Parental influences on youth involvement in sports. In M. R. Weiss (Ed.), Developmental sport and exercise psychology: A lifespan perspective (pp. 145-164). Champaign, IL: Human Kinetics.

Giorgi, A. (2009). The descriptive phenomenological method in psychology: A modified husserlian approach. Pittsburgh, PA: Duquesne University Press.

Giorgi, A., \& Giorgi, B. (2008a). Phenomenological psychology. In C. Willig, \& W. Stainton-Rogers (Eds.), Handbook of qualitative research in psychology (pp. 165178). London: Sage Publications Ltd.

Giorgi, A., \& Giorgi, B. (2008b). Phenomenology. In J. A. Smith (Ed.), Qualitative psychology: A practical guide to research methods (2nd ed., pp. 26-52). London: Sage Publications Ltd.

Gold, R.L. (1958). Roles in sociological field observations. Social Forces, 36, 217-223.

Gould, D., Lauer, L., Rolo, C., Jannes, C., \& Pennisi, N. (2008). The role of parents in tennis success: Focus group interviews with junior coaches. The Sport Psychologist, 22(1), $18-37$.

Harwood, C., Drew, A., \& Knight, C. (2010). Parental stressors in professional youth football academies: A qualitative investigation of specializing stage parents. Qualitative 
Harwood, C., \& Knight, C. (2009a). Stress in youth sport: A developmental investigation of tennis parents. Psychology of Sport and Exercise, 10(4), 447-456. doi: 10.1016/j.psychsport.2009.01.005

Harwood, C., \& Knight, C. (2009b). Understanding parental stressors: An investigation of british tennis-parents. Journal of Sports Sciences, 27(4), 339-351. doi: $10.1080 / 02640410802603871$

751

752

753

754

755

756

757

758

759

760

761

762

763

764

765

766

767

768

Henwood, K., \& Procter, J. (2003). The 'good father': Reading men's accounts of paternal involvement during the transition to first-time fatherhood. British Journal of Social Psychology, 42(3), 337-355. doi: 10.1348/014466603322438198

Holt, N., Tamminen, K. A., Black, D. E., Sehn, Z. L., \& Wall, M. P. (2008). Parental involvement in competitive youth sport settings. Psychology of Sport and Exercise, 9(5), 663-685. doi: 10.1016/j.psychsport.2007.08.001

Kay, T. (2009). Fathers and sons: being ‘father angel’. In Kay, T (Ed.), Fathering Through Sport and Leisure (pp. 106-123). Oxon: Routledge

Kerr, G. A., \& Stirling, A. E. (2012). Parents’ reflections on their child's experiences of emotionally abusive coaching practices. Journal of Applied Sport Psychology, 24(2), 191-206. doi: 10.1080/10413200.2011.608413

Knight, C. J., \& Holt, N. L. (2013). Factors that influence parents' experiences at junior tennis tournaments and suggestions for improvement. Sport, Exercise, and Performance Psychology, 2(3), 173-189.

Lally, P., \& Kerr, G. (2008). The effects of athlete retirement on parents. Journal of Applied Sport Psychology, 20(1), 42-56. doi:10.1080/10413200701788172

Langdridge, D. (2007). Phenomenological psychology: Theory, research and method. Harlow, Essex: Pearson Education Ltd. 
769 Lauer, L., Gould, D., Roman, N., \& Pierce, M. (2010a). How parents influence junior tennis

770

771

772

773

774

775

776

777

778

779

780

781

782

783

784

785

786

787

788

789

790

791

792

793 players development: Qualitative narratives. Journal of Clinical Sport Psychology, 4(1), 69-92.

Lauer, L., Gould, D., Roman, N., \& Pierce, M. (2010b). Parental behaviors that affect junior tennis player development. Psychology of Sport and Exercise, 11(6), 487-496. doi: 10.1016/j.psychsport.2010.06.008

Merleau-Ponty, M. (1962). Phenomenology of perception (C. Smith, Trans). London: Routledge \& Kegan Paul. (Original work published 1945).

Moustakas, C. (1994). Phenomenological research methods. Thousand Oaks, CA: Sage Publications Ltd.

Norlyk, A., \& Harder, I. (2010). What makes a phenomenological study phenomenological? An analysis of peer-reviewed empirical nursing studies. Qualitative Health Research, 20(3), 420-431. doi: 10.1177/1049732309357435

O'Connell, D. C., \& Kowal, S. (1999). Transcription and the issue of standardization. Journal of Psycholinguistic Research, 28(2), 103-120.

Omli, J., \& LaVoi, N. M. (2012). Emotional experiences of youth sport parents I: Anger. Journal of Applied Sport Psychology, 24(1), 10-25. doi: $10.1080 / 10413200.2011 .578102$

O'Rourke, D. J., Smith, R. E., Smoll, F. L., \& Cumming, S. P. (2011). Trait anxiety in young athletes as a function of parental pressure and motivational climate: Is parental pressure always harmful? Journal of Applied Sport Psychology, 23(4), 398-412.

Sparkes, A. C., \& Smith, B. (2009). Judging the quality of qualitative inquiry: Criteriology and relativism in action. Psychology of Sport and Exercise, 10(5), 491-497. doi: 10.1016/j.psychsport.2009.02.006

The Premier League. (2011). Elite player performance plan. 
794 Tracy, S. J. (2010). Qualitative quality: Eight "big-tent" criteria for excellent qualitative research. Qualitative Inquiry, 16(10), 837-851. doi: 10.1177/1077800410383121

796

797

798

799

800

801

802

803

804

805

806

807

808

809

810

van Manen, M. (1990). Researching lived experience: Human science for an action sensitive pedagogy. New York: State University of New York Press.

Wiersma, L. D., \& Fifer, A. M. (2008). "The schedule has been tough but we think it's worth it": The joys, challenges, and recommendations of youth sport parents. Journal of Leisure Research, 40(4), 505-530.

Wippert, P., \& Wippert, J. (2010). The effects of involuntary athletic career termination on psychological distress. Journal of Clinical Sport Psychology, 4(2), 133-149.

Wolfenden, L. E., \& Holt, N. L. (2005). Talent development in elite junior tennis: Perceptions of players, parents, and coaches. Journal of Applied Sport Psychology, 17(2), 108-126. doi: 10.1080/10413200590932416

Wuerth, S., Lee, M. J., \& Alfermann, D. (2004). Parental involvement and athletes' career in youth sport. Psychology of Sport and Exercise, 5(1), 21-33. doi: 10.1016/S14690292(02)00047-X

Yardley, L. (2000). Dilemmas in qualitative health research. Psychology \& Health, 15(2), 215-228. doi: 10.1080/08870440008400302 